\title{
Aprender para comunicar; bases de partida en entornos de humedales ibéricos.
}

\author{
Gema SÁNCHEZ EMETERIO \\ Universidad de Castilla-La Mancha \\ Gema.Sanchez@uclm.es \\ Beatriz GARCÍA FERNÁNDEZ \\ Universidad de Castilla-La Mancha \\ Beatriz.García@uclm.es
}

\section{Resumen}

En este artículo se presenta un estudio realizado con alumnos de las Facultades de Educación de Faro (Portugal), Ciudad Real y Toledo (España), situadas cerca de humedales protegidos, relativo al conocimiento y percepción de estos espacios. Los resultados muestran que, generalmente, los alumnos tienen vagas nociones sobre el funcionamiento, valores y funciones de los humedales antes de trabajar con ellos. Tras el trabajo en el aula este hecho cambia, aunque persisten carencias en la compresión de aspectos relevantes que deberían comunicar en las aulas en su futuro ejercicio docente.

Palabras clave: Comunicación, Facultades de Educación, Humedales ibéricos, Aprendizaje Significativo Sostenible, Evaluación.

\section{Learning to communicate; baselines in Iberian wetland environments.}

\begin{abstract}
A study focused on the perception and knowledge of wetlands among the students of the Faculties of Education in Faro (Portugal), Ciudad Real and Toledo (Spain), located near protected wetlands, is presented in this article. The results show that, in general, students have vague ideas about the functioning, values and functions of wetlands before working on them. This fact changes after their work in the classroom, although a lack of understanding of important issues that they should communicate in the classroom in their teaching function remains.
\end{abstract}

Key Words: Communication, Faculties of Education, Iberian Wetlands, Sustainable Meaningful Learning, Assessment.

Referencia normalizada:

Sánchez Emeterio, G. y García Fernández, B. (2013) Aprender para comunicar; bases de partida en entornos de humedales ibéricos. Historia y Comunicación Social. Vol. 18. № Especial Noviembre. Págs. 777-788.

Sumario 1. Introducción 2. Área de estudio 3. Relevancia de los humedales 3.1 Relevancia geositémica 3.2 Relevancia didáctica 4. Metodología 5. Resultados 6. Conclusiones. 7. Referencias bibliográficas 


\section{Introducción}

El Sistema Educativo en España, lejos de basarse en reformas sobre eficacia competitiva, necesita basarse en aprendizajes profundos, relevantes y duraderos. Los docentes deben desarrollar competencias sobre los problemas ambientales y capacidad de comunicación, para educar a ciudadanos responsables que contribuyan a mejorar el medio que les rodea, y no conocimientos desvinculados de las repercusiones sobre los espacios que habitan. El aprendizaje significativo sostenible (Galagovsky, 2004) y las Tecnologías de la Información y la Comunicación pueden facilitar el desarrollo de competencias y estrategias cognitivas para lograr involucrar a un grupo de alumnos en un aprendizaje profundo. Para conseguir que el aprendizaje sea significativo, relevante y sostenible es necesario un análisis profundo sobre los espacios, pero también sobre los conocimientos previos de los alumnos y la forma de comunicar los contenidos.

Los humedales son los ecosistemas más productivos del planeta (Mitchell, 1984; Owen y Black, 1990; Ellis y Wang, 1997; Chen y Lu, 2003). A pesar de ello, durante el siglo pasado y especialmente en la segunda mitad del mismo, desapareció aproximadamente el $60 \%$ de la superficie ocupada por lagos y humedales en España (Casado y Montes, 1995). Los alumnos de entornos ricos en humedales desconocen en una amplia mayoría, tal y como muestran los resultados de este estudio, la mera existencia de estos geosistemas, su alto grado de degradación, la relevancia de sus valores y funciones y las nociones básicas sobre su funcionamiento.

Pese a la importancia que está adquiriendo la Educación Ambiental en humedales y la puesta en marcha de programas educativos y de sensibilización desarrollados por organismos públicos (Confederaciones Hidrográficas o Ministerio de Agricultura Alimentación y Medioambiente) y privados (por ejemplo, la Fundación Global Nature) en las aulas, los futuros docentes se centran sólo en los aspectos aislados más llamativos de los humedales más conocidos, sin considerar el funcionamiento de forma holística del humedal ni el de la Red de Humedales en la que se inserta.

Con la finalidad de conocer las diferencias de percepción y conocimiento de los humedales entre los estudiantes, se ha realizado un estudio en tres Facultades de Educación situadas en Espacios Naturales Protegidos de reconocida relevancia internacional, además de ejes motores ambientales y económicos de sus entornos: La Ría Formosa, en Portugal, y la Reserva de la Biosfera de la Mancha Húmeda (RBMH), en España. Se ha encuestado a una muestra de estudiantes de las Facultades de Educación de Toledo (FET) y Ciudad Real (FECR), de la Universidad de Castilla-La Mancha (UCLM), y de la Facultad de Ciencias de la Educación y la Comunicación de la Universidad del Algarve (UALG), en Faro. Concretamente se ha encuestado a alumnos de segundo (que no han estudiado humedales) y cuarto curso de Educación Primaria (tras trabajar los humedales en clase) en cada Facultad. 


\section{2. Área de estudio}

Las áreas en las que se ha llevado a cabo este trabajo comparten muchas similitudes, destacando para el objeto de este estudio, sus humedales de relevancia internacional. En Faro, en el Algarve, está la Ría Formosa (Fig. 1); una de las siete maravillas de Portugal, incluida en la lista de humedales de interés mundial definido por la Convención de Ramsar. Declarada Parque Natural en 1987, abarca una superficie de unas 18.000 ha. Es considerada un área importante de aves (IBA) y es parte de la red Natura 2000. En este geosistema se encuentran, entre una gran riqueza biológica, especies en peligro de extinción como el camaleón, o la mayor población de caballitos de mar del mundo. Además, la ría constituye una importante área socioeconómica, relevante por el marisqueo, el turismo y las actividades recreativas.

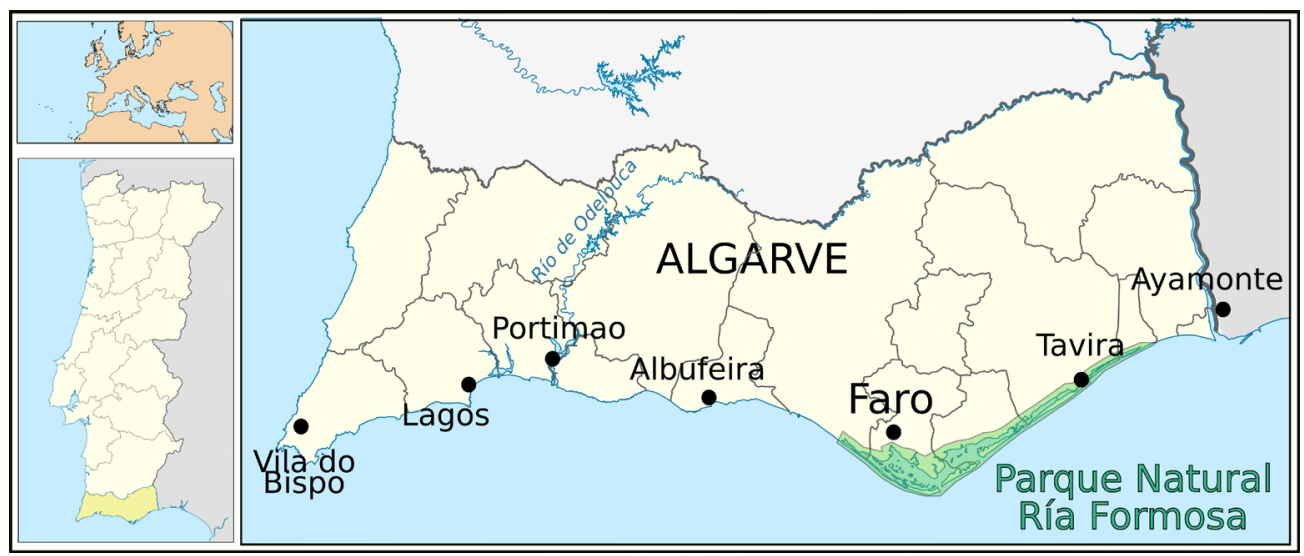

Figura 1. Situación del Parque Natural de la Ría Formosa.

La Ría Formosa es una laguna costera única que cambia constantemente debido al continuo movimiento de vientos, corrientes y mareas. Las lagunas de La Mancha, al ser sistemas lénticos, cambian principalmente dependiendo de las precipitaciones y/o aportes de aguas subterráneas, proporcionando gran diversidad de hábitats, entre otros bienes y valores. La RBMH (Fig. 2), declarada en 1981 para conservar las lagunas que la conforman respetando sus valores culturales y patrimoniales (García del Castillo et al., 2011), abarca una superficie de unas 25.000 ha. Según Constanza et al. (1997) el valor productivo de las rías es el mayor del planeta, seguido de los humedales, por encima de los pastizales, bosques, lagos, ríos, etc., con ratios de producción que pueden exceder los de ecosistemas terrestres (Turner, 1977). $\mathrm{He}$ aquí otras razones de peso para ser considerados espacios de gran relevancia didáctica en entorno en el que se insertan; especialmente cumplen uno de los principales prerrequisitos para lograr un aprendizaje significativo: el contexto pertinente (Fyrenius, 2005), percibiendo los contenidos como relevantes en escenarios reales, lo cual resulta motivador. 


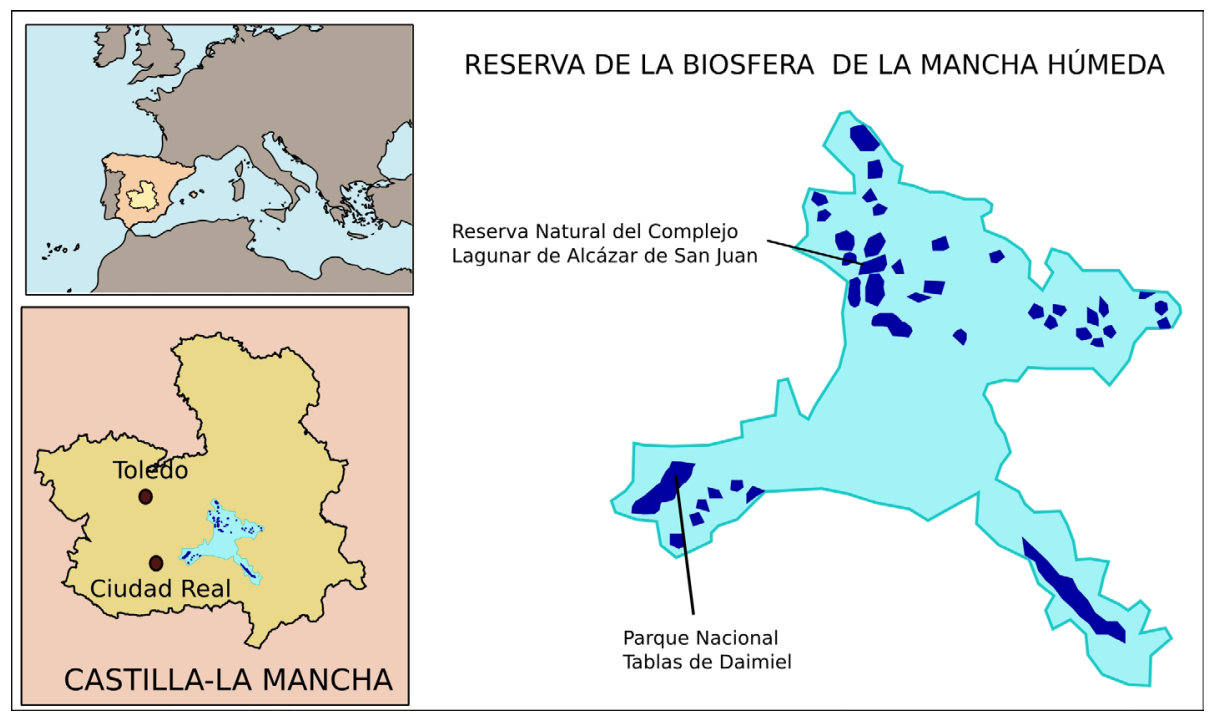

Figura 2. Situación de la Reserva de la Biosfera de La Mancha Húmeda y sus principales lagunas.

\section{Relevancia de los humedales}

\subsection{Relevancia geosistémica}

Los humedales ocupan sólo un $6 \%$ de la superficie terrestre pero proporcionan importantes recursos que contribuyen al mantenimiento de la biodiversidad. Por ejemplo, mitigan las inundaciones, protegen las áreas costeras de las tormentas, provén de agua de calidad, recargan los acuíferos, sirven de fuentes y sumideros, transforman materiales y producen comida y otros bienes para abastecimiento humano (Cherry, 2012).

Merece la pena detenerse en la relevancia del funcionamiento ecosistémico integral, sin supravalorar sólo uno de los componentes del sistema, junto a la relevancia de considerar que los humedales son un conjunto de sistemas entre los cuales se establecen numerosas relaciones (flujos de agua, dinámicas metapoblacionales, etc.). Por tanto, su comprensión real sólo puede entenderse de forma holística, tanto dentro del humedal como en la Red en que se integra. Contrastar las tendencias de humedales en una determinada zona de estudio con las tendencias observadas en otros humedales del entorno permite diferenciar entre las tendencias propias de un humedal o las tendencias globales (Figerola y Green, 2003) y comprender así el funcionamiento global real que permita su conservación y llevar a cabo en ellos actuaciones destinadas a mitigar los daños existentes. Estos daños son notables en muchos de los ecosistemas de la RBMH, como ocurre en el Parque Nacional de Las Tablas de Daimiel (PNTD) (Fig. 2). Este ecosistema, cuya problemática ha sido ampliamente tratada 
por los medios de comunicación, se encuentra desde los años ochenta desconectado del acuífero regional, fuente de uno de sus principales aportes hídricos. Sin embargo, el hecho de que el acuífero pueda volver a aportar agua de acuerdo a las actuales políticas de extracción (Yustres et al., 2013) y la rápida respuesta del Parque ante aportes externos de agua en situaciones de largas sequías (Navarro et al., 2011; Navarro et al., 2012; García Fernández et al., 2013) hace que las expectativas de restauración de este ecosistema sean muy elevadas, como también lo son su funcionamiento e interrelaciones con otros elementos del geosistema. El PNTD es sólo un ejemplo de estos ecosistemas que, aunque deteriorados, responden positivamente a estrategias de restauración, lo que hace que cobre aún más sentido sensibilizar y concienciar a las futuras generaciones de su relevancia desde una educación apoyada en bases científicas, fomentando su conservación y restauración a medio y a largo plazo con el fin de evitar los desastres derivados de la gestión sucedidos en años anteriores.

Formar ciudadanos capaces de comprender el funcionamiento real de estos ecosistemas, y por tanto, de plantear soluciones que mitiguen los importantes daños existentes, implica comunicar a los estudiantes una visión holística, alejada de cualquier visión simplista, reduccionista o centrada en aspectos estéticos en el peor de los casos de estas realidades, y que debe ser elaborada a partir de conocimientos científicos. Aunar esfuerzos con el objetivo de transponer las investigaciones científicas al aula e investigar paralelamente en cuestiones didácticas relacionadas con las científicas, unido a la sinergia de hacerlo llegar a los alumnos mediante metodologías y medios de comunicación que les resulten motivadores y significativos, hará que seamos más eficientes en investigación, y también que el proceso de comunicación en el aprendizaje sea más eficaz (García Fernández et al., 2012).

\subsection{Relevancia didáctica}

La RBHM cuenta con rico patrimonio de humedales en una zona semiárida, cuya importancia suele ser ajena a muchos estudiantes, y a veces, profesores, hecho que contrasta con que estos geosistemas constituyan un importante recurso didáctico para la consolidación de competencias. En la legislación vigente en España en materia educativa en Educación Primaria, el Conocimiento del Medio tiene un papel destacado. Si bien la ciencia se basa en la observación, los científicos no tienen acceso directo a la mayoría de los fenómenos naturales (Lederman, 2002), y por tanto no pueden poner en contacto a sus alumnos con el medio. El desarrollo del conocimiento científico implica realizar observaciones en la naturaleza, y los futuros docentes, en este caso, sí tienen a su disposición ese medio en el que observar directamente fenómenos naturales.

Además de las bases metodológicas y científicas, algunas bases pedagógicas, como las de Rousseau (1763), destacan la importancia de partir de conocimiento de lo cercano, ya que según este pedagogo nacemos sensibles, y desde nuestro nacimiento excitan en nosotros diversas impresiones los objetos que nos rodean. Estas bases avalan la viabilidad de trabajar el conocimiento científico y los contenidos reflejados en el currículo de Primaria a partir de los humedales. De hecho, hablar de 
aprendizaje significativo implica que el elemento central del proceso de enseñanza y aprendizaje es la construcción de significados propios por parte del alumno (Coll, 1988), y el contacto con el medio objeto de estudio es un elemento fundamental para llevar a cabo con éxito este proceso. Además, los humedales cumplen uno de los principales prerrequisitos para lograr un aprendizaje significativo; el contexto pertinente (Fyrenius, 2005), percibiendo los contenidos como relevantes en escenarios reales, lo cual resulta motivador.

La ciencia nunca comienza con observaciones neutrales (Popper, 1992); por eso la visión de los conocimientos previos de los alumnos es clave al ser son determinantes en el desarrollo de los procesos de enseñanza-aprendizaje. Las evaluaciones iniciales y finales son indicadores del estado de conocimiento de los futuros docentes. Además, realizar una evaluación de los logros obtenidos permite desarrollar estrategias de mejora para optimizar los resultados en futuros cursos académicos.

\section{Metodología}

Se ha realizado un cuestionario a un total de 82 estudiantes, 37 de los cuales pertenecen a la UALG, y 45 a la FET y a la FECR. Este cuestionario ha sido utilizado como evaluación inicial para aquellos estudiantes que no habían trabajado previamente con humedales, y como evaluación final en estudiantes que sí habían trabajado estos ecosistemas. El objetivo de la realización de este cuestionario ha sido conocer las diferencias de percepción y conocimiento entre los estudiantes que habían trabajado humedales previamente y los que no lo habían hecho, partiendo de los conceptos y procesos relevantes en los humedales y los contenidos y competencias a desarrollar en los Decretos de Educación. El cuestionario (Fig. 3) fue validado mediante juicio de expertos, sometiendo cada ítem a un proceso de revisión de contenido, semántica y sintáctica.

1. Escribe cinco palabras sobre los conceptos que creas que están más relacionados con los humedales.

2. Rodea con un círculo las respuestas que consideres correctas.

a) ¿Consideras que es normal que los humedales mediterráneos se sequen?

SI NO

b) ¿Consideras que los humedales que se secan en verano tienen menor valor ecológico que aquellos que permanecen siempre encharcados?

SI NO

c) ¿En humedales de interior podemos encontrar especies de flora y fauna semejantes a aquellas

características de zonas estuario - costeras?

d) ¿Existen zonas húmedas de interior con salinidad mayor que la del agua del mar?

e) ¿Consideras los humedales como un recurso didáctico relevante?

f) ¿Los humedales aportan elementos positivos para los núcleos urbanos próximos?

Escribe algunos valores y funciones de los humedales; especialmente aquellos que puedan trabajarse en educación.

Valores:

Funciones:

4. Señala, del 1 al 10 , cuánto crees que sabes sobre humedales.

$\begin{array}{llllllllll}1 & 2 & 3 & 4 & 5 & 6 & 7 & 8 & 9 & 10\end{array}$

Figura 3: Cuestionario. 
Los alumnos que habían trabajado humedales previamente, un total de 37, pertenecían a la FET y la FECR, de ahora en adelante muestra 1 (M1). Los alumnos que no habían trabajado con humedales, un total de 45 , y a partir de ahora muestra 2 (M2) pertenecían a la UALG (37 alumnos) y a la UCLM ( 8 alumnos).

La metodología de trabajo en el aula con la M1 consistió en Aprendizaje Basado en Problemas mediante trabajo colaborativo, organización del ambiente de aprendizaje que mejor responde a los requisitos del aprendizaje significativo (Margetson, 1993). Esta metodología permite al alumnado trabajar mediante la exploración de sus conocimientos previos y decidir a partir de este punto, qué información necesitan adquirir para resolver la situación que se les ha propuesto trabajar (Savin-Baden, 2000), de ahí la importancia de evaluar los conocimientos previos, ya que el alumno construye personalmente un significado sobre la base de los significados que ha podido construir previamente (Miras, 2002). Cada grupo de trabajo realizó una tarea de investigación enfocada a diferentes aspectos de la $\mathrm{RBMH}$, como el régimen hídrico natural y alteraciones antropogénicas, flora y su relación con el régimen hídrico y el sustrato, fauna y su relación con el recursos disponibles, ciclo hidrológico, efectos del cambio climático, salinidad y formación de costra salina, estado ecológico, contaminación, propuestas de mejora, geomorfología, humedales como parte del patrimonio y figuras de protección. También se realizaron aplicaciones didácticas de los humedales para Educación Primaria desde diferentes enfoques anteriores, para finalmente llevar a cabo una puesta en común, con el objetivo de proporcionar una visión holística del funcionamiento geosistémico.

\section{Resultados}

En la primera pregunta, los alumnos de la M1 nombran una media de 3,6 conceptos correctos relacionados con humedales, frente a 1,9 de la M2. Los más citados por la M1 fueron: flora, fauna, agua, migración de aves, vida, patrimonio y conservación, mientras que los más citados por la M2 fueron flora, fauna y agua, conceptos menos específicos que los anteriores.

Tan sólo un $31 \%$ de la M1 responde que es normal que los humedales se sequen en verano, frente a $51 \%$ de la M2. Es de destacar que $19 \%$ de los alumnos de la M2 no responde a esta pregunta. Pese a haber estudiado estos temas en el aula, los estudiantes de la M1 aún tienen estos conceptos erróneos arraigados. Puede deberse en parte a que la M1 ha realizado su trabajo de investigación y aplicación didáctica sobre el Complejo Lagunar de Alcázar de San Juan (Fig. 2); lagunas con niveles hídricos alterados antropogénicamente, manteniendo una lámina de agua permanente durante todas las épocas del año, incluida la estival. El estudio de estos procesos hídricos no parece haber repercutido en su comprensión, por lo que es necesario realizar mayor énfasis en el tratamiento de estos conceptos. 
El $89 \%$ de la M1 y un 46\% de la M2 respondió que los humedales que se secan en verano no tienen menor valor ecológico que aquellos que no lo hacen, lo que indica que el estudio de los humedales ha fomentado la puesta en valor de estos geosistemas y que entiendan como natural el proceso de secado estival, y no como un signo de deterioro o de menor valor ambiental. Sin embargo, la mitad de la M2 no cree que un humedal que se seca en verano pueda tener el mismo valor ecológico que aquellos que sí lo hacen, hecho que puede deberse a que gran parte de esta muestra ha estado siempre en contacto con la Ría Formosa, con procesos de inundación y desecación muy laxos, asociados a mareas, y no han estudiado otras zonas húmedas además de la Ría en ninguna de sus etapas educativas. Por tanto, el desconocimiento de otros funcionamientos ecosistémicos diferentes hace que no los valoren adecuadamente.

Un $71 \%$ de la M1 y un $72 \%$ de la M2 es incapaz de relacionar la avifauna presente en humedales de interior y costeros, de lo que se desprende una necesidad de trabajar los fenómenos migratorios de aves entre diferentes humedales, así como realizar un cambio de estrategia metodológica que permita adquirir de un modo más profundo estos conceptos.

Cuando se les pidió indicar si existían zonas húmedas de interior con mayor salinidad que la del agua del mar, un $63 \%$ de la M1 respondió afirmativamente, frente a un $33 \%$ de la M2. Las investigadoras, mediante el método del observador participante, obtuvieron impresiones de los estudiantes acerca de los conocimientos aprendidos en el aula. La gran salinidad de las aguas de muchos de los humedales que conforman la RBMH fue uno de los aspectos que más llamó la atención a los estudiantes, puesto que no conocían el concepto de endorreísmo y no habían reflexionado acerca de los efectos que este fenómeno podía tener en el medio físico, y por tanto, en la flora y la fauna. Los alumnos de la M2 no tienen humedales de interior cercanos, ni han trabajado el concepto de cuenca endorreica. Es fundamental que este concepto se trabaje de manera conjunta con las dinámicas migratorias de las aves y con la vegetación existente en humedales costeros y endorreicos, para poder comprender de una manera holística el funcionamiento ecosistémico.

Un 98\% de la M1 considera los humedales como recurso didáctico relevante, frente a un 57\% de la M2, debido a que en la UCLM se ha trabajado este aspecto, aplicando los humedales al Decreto 68/2007 que regula la Educación Primaria en Castilla-La Mancha, elaborando actividades y programaciones didácticas para los distintos ciclos y cursos que la componen.

El 98\% de la M1 responde que los humedales aportan elementos positivos a núcleos urbanos próximos, frente a un $66 \%$ de la M2, compuesta fundamentalmente por estudiantes de la UALG. Pese a la importancia de la Ría Formosa en la historia de la actividad económica de Faro como lugar de pesca, como ecosistema y como atractivo turístico, los estudiantes no la identifican masivamente como un ecosistema que aporte elementos positivos al núcleo urbano, lo que nace necesario trabajar en la puesta en valor de estos ecosistemas. 
Se les pidió a los estudiantes que enumeraran valores y funciones de los humedales, en especial aquellos que pudieran trabajarse en educación. Un 76\% de la M1 es capaz de citar algún valor, frente a un $16 \%$ en la M2. En el caso de las funciones, un $50 \%$ de la M1 es capaz de citar alguna de ellas, frente al 7\% de la M2. Es muy destacable que, dentro de este 7\% no haya ningún estudiante que cite ninguna de las importantes funciones de la Ría Formosa, pese a que es un importante motor de la economía de la localidad. Esta diferencia se debe a que en los grupos en que se han trabajado humedales se ha hecho hincapié en sus valores y funciones, diferenciando ambas, con el fin de poner en valor estos ecosistemas, puesto que aquello que no se conoce, no se valora y no se conserva. Y no hay que olvidar la responsabilidad de los futuros maestros como educadores de las futuras generaciones de ciudadanos.

La última pregunta versa sobre la percepción de los estudiantes sobre el conocimiento que poseen sobre humedales en una escala Likert del 1 al 10, donde 1 significa que no conocen absolutamente nada y 10 que sus conocimientos al respecto son muy amplios. En cuanto a la percepción sobre el conocimiento que poseen acerca de los humedales, los estudiantes de la M1 tienen una mayor percepción puntuándolo con un 5 sobre 10 frente a un 3,7 de la M2. Ambos resultados indican que los estudiantes son conscientes, incluso aquellos que han trabajado con humedales, de que su grado de conocimiento no es demasiado elevado.

\section{Conclusiones}

Los resultados muestran que prácticamente todos los alumnos encuestados, futuros docentes, desconocen el funcionamiento real de los humedales. Muchos no conocen los elementos clave y algunos desconocen su mera existencia. A pesar de ello, una amplia mayoría reconoce el valor de emplearlo como recurso didáctico. Por tanto, la principal conclusión que se extrae, una vez justificada la relevancia didáctica, es que los humedales deben ser más estudiados en el aula, ya que aún en el caso en que han sido estudiados, su funcionamiento ecosistémico resulta de difícil comprensión.

Los alumnos que sí han estudiado los humedales, además de tener un mayor conocimiento, tienen una visión más amplia y holística, de los humedales, y por lo tanto, más real. No los reducen sólo a los aspectos más conocidos y llamativos; de agua, plantas y aves, sino que además los perciben como elementos patrimoniales de especial relevancia. Y como un recurso didáctico importante, al emplear conceptos como, por ejemplo, el de ciclo del agua.

Aun así, hay vacíos en la comprensión de estos geosistemas, especialmente en lo referente a los procesos hídricos y la similitud de funcionamiento de los distintos tipos de sistemas de humedales (ría-lagunas) y sus interrelaciones, ya que, como se desprende del estudio realizado, a pesar de conocer la salinidad, las aves y los requerimientos de estas aves, no son capaces de establecer conexiones entre dos geosistemas. Por tanto, es necesario incidir en la comprensión holística y el establecimiento 
de interrelaciones, mejorando el proceso de comunicación entre los distintos grupos (que trabajan en profundidad aspectos concretos) de alumnos y entre estos y sus profesores, para que el proceso de Enseñanza-Aprendizaje sea más efectivo y el nivel de adquisición de competencias sobre el medio en que se desenvuelven sea más elevado.

Al igual que se desprende de este trabajo, en estudios anteriores elaborados en torno a la zona de estudio de la parte española, el profesorado considera que el grado de conocimiento de los alumnos de Geología y Geografía física, sobre su entorno más inmediato es, generalmente escaso (Carricondo, 2010). En el estudio de Carricondo también se concluye que los alumnos tienen poco conocimiento y escasa conciencia de la relevancia del entorno físico inmediato, y se apunta como principal causa la ausencia de materiales, ya que el profesorado es el encargado de elaborarlo, y en numerosas ocasiones, también son desconocedores. En el caso de los humedales, los alumnos que sí los han trabajado los ven como un valioso recurso ya que parte de su trabajo en el aula consistió en la realización de materiales didácticos, debido a que una de las principales tareas de las Facultades de Educación debe ser la elaboración de materiales didácticos como recursos que permitan el conocimiento de los elementos relevantes del Medio. En este sentido se ha de destacar que los alumnos de la UALG que no han estudiado humedales, cuentan con un valioso recurso didáctico en el entorno de la Ría Formosa: El Centro de Ciencia Viva, espacio dedicado al conocimiento científico de forma didáctica sobre la Ría, además del propio humedal y numerosas excursiones guiadas por el mismo. Pese a esto y aunque los alumnos asocian a la Ría conceptos como flora, fauna, migración, agua y ecosistema, estos no han llevado a cabo una reflexión acerca de cómo estos conceptos pueden ser llevados a las aulas de Primaria del Algarve, al contrario que ocurre entre los alumnos de la UCLM donde los encuestados son conscientes del potencial de estos ecosistemas como recurso didáctico.

\section{Referencias bibliográficas}

CARRICONDO SÁNCHEZ, J.F. (2010). La provincia de Ciudad Real como recurso didáctico en el campo de la Geología en la Etapa de Educación Secundaria. Valoración de la actividad didáctica desarrollada en los museos y centros de interpretación. Granada: Facultad de Ciencias de la Educación de la Universidad de Granada. Tesis Doctoral

CASADO DE OTAOLA, S.; MONTES DEL OLMO, C. (1995). Guía de los lagos y humedales de España. Madrid: J. M. Reyero (ed.).

CHEN Y.; LU, X. (2003). "The wetland function and research tendency of wetland science". En: Wetland Science, 1, p. 7-10.

CHERRY, J. A. (2012). "Ecology of Wetland Ecosystems: Water, Substrate, and Life." En: Nature Education Knowledge, 3 (10):16. 
COLL, C. (1988). "Significado y sentido en el aprendizaje escolar. Reflexiones en torno al concepto de aprendizaje significativo" En: Infancia y aprendizaje, 41, p. 131-142.

CONSTANZA, R.; D'ARGE, R.; DE GROOT, R.; FARBER, S.; HANNON, B.; LIMBURG, K.; NAEEM, S.; PARUELO, J.; RASKIN, R. G.; SUTTON, P.; VAN DER BELT, M. (1997). "The value of the world's ecosystem services and natural capital." En: Nature, 387, p. 253-260.

ELLIS, E.C.; WANG, S.M. (1997). "Sustainable Traditional Agriculture in the Tai Lake Region of China." En: Agriculture, Ecosystems \& Environment, 61 (2-3), p. 177-193.

FIGUEROLA, J.; GREEN, A. (2003). "Aves acuáticas como bioindicadores en los humedales." Ecología, manejo y conservación de los humedales. Almería: Instituto de Estudios Almerienses. P. 47-60.

FYRENIUS, A.; BERGDAHL, B.; SILÉN C. (2005). "Lectures in problem-based learning-Why, when and how? An example of interactive lecturing that stimulates meaningful learning." En: Medical Teacher, 27 (1), p. 61-65.

GARCÍA DEL CASTILLO CRESPO, J.; RUBIO GARCÍA, M.A.; LÓPEZ BRAVO, A. (Coord.) (2011). Reserva de la Biosfera de la Mancha Húmeda: retos y oportunidades de futuro. Toledo: Dirección General de Áreas Protegidas y biodiversidad, Junta de Comunidades de Castilla-La Mancha.

GARCÍA FERNÁNDEZ, B.; NAVARRO GÁMIR, V.; ASENSIO SÁNCHEZ, L. (2013). "A synthetic model of surface inundation relationships and water source impacts of the wetland Las Tablas de Daimiel National Park, Central Spain". En: Ecological Engineering, 61, p. 407-418.

GARCÍA FERNÂNDEZ, B.; SANCHEZ EMETERIO, G.; SÁNCHEZ RAMOS, D. (2012). Transposición didáctica y comunicación: el estudio de los humedales. En: Estudios sobre el Mensaje Periodístico, 18, p. 347-356.

LEDERMAN, N. G; ABD-EL-KHALICK A., F.; BELL, R. L., SCHWARTZ, R. S. (2002). "Views of Nature of Science Questionnaire: Toward Valid and Meaningful Assessment of Learners' Conceptions of Nature of Science." En: Journal of Research in Science Teaching, 39 (6), p. 497-52.

MARGETSON, D. (1993). Understanding problem-based learning, Educational Philosophy and Theory. P. 40-57.

MIRAS, M. (1999). "Un punto de partida para el aprendizaje de nuevos contenidos: Los conocimientos previos". En COLL, C.; MARTÍN, E.; MAURI, T.; MIRAS, M.; ONRUBIA, J.; SOLÉ, I.; ZABALA, A. (2002). El constructivismo en el aula. Barcelona: Editorial Graó.

MITCHELL, R. (1984). "The ecological basis for comparative primary production". En: LOWRANCE, R.; STINNER, B.R.; HOUSE G.J. (Editors). Agricultural Ecosystems: Unifying Concepts. New York: John Wiley and Sons.

NAVARRO, V.; GARCIA, B.; ASENSIO, L. (2012). "Characterization of the infiltration rate in Las Tablas de Daimiel National Park, Central Spain". En: Hydrological Processes, 26 (3), p. 367-378. 
NAVARRO ,V.; GARCÍA, B.; SÁNCHEZ, D.; ASENSIO, L. (2011). “An evaluation of the application of treated sewage effluents in Las Tablas de Daimiel National Park, Central Spain." En: Journal of Hydrology, 401 (1-2), p. 53-64.

OWEN M.; BLACK, J.M. (1990). Waterfowl Ecology. Tertiary level biology. Blackie. New York :Chapman and Hall.

POPPER, K.R. (1992). The logic of scientific discovery. London: Routledge. (Original publicado en 1934).

ROUSSEAU, J. J. (1763). Emilio. México, D.F. Dirección General de Publicaciones, 1975. México.

SAVIN-BADEN, M. (2000). Problem-based Learning in Higher Education: Untold Stories. The Society for Research into Higher Education \& Open University Press. Suffolk: St Edmundsbury Press, Bury St Edmunds.

TURNER, R. E. (1977). "Intertidal vegetation and commercial yields of penaeid shrimp". En: Transactions of the American Fisheries Society, 106, p. 411-416.

YUSTRES, A.; NAVARRO, V.; ASENSIO, L.; CANDEL, M.; GARCÍA, B. (2013). "Groundwater resources in the Upper Guadiana Basin (Spain): a regional modelling analysis.” En: Hydrogeology Journal, 21(5), p 1129-1146.

\section{Las autoras}

Gema Sánchez Emeterio. Licenciada en Geografía. DEA en Territorio, Infraestructuras y Medioambiente. Programa en el que actualmente cursa estudios de doctorado. Profesora Ayudante de Didáctica de las Ciencias Sociales. Facultad de Educación de Toledo. Departamento de Geografía y Ordenación del Territorio. Universidad de Castilla-La Mancha. Miembro del grupo de investigación en hidroecología del Centro Regional del Agua de Castilla-La Mancha. Áreas de investigación y docencia: Geografía (general), Didáctica de las Ciencias Sociales (Facultad de Educación y Máster de formación de Profesorado de Enseñanza Secundaria) y humedales (desde el punto de vista de la Geografía, ecología y didáctica), Cooperación al Desarrollo (tecnologías del agua y regionalismos).

Beatriz García Fernández. Doctora Ingeniero de Caminos, Canales y Puertos. Profesora del Área de Didáctica de las Ciencias Experimentales en la Facultad de Educación de Ciudad Real. Adscrita al departamento de Pedagogía de la Universidad de Castilla-La Mancha. Docencia en la Facultad de Educación de Ciudad Real dentro del Grado de Maestro de Educación Primaria. Áreas de investigación: Didáctica de las Ciencias Experimentales, Ingeniería ecológica en humedales, aplicaciones didácticas de humedales en Educación Primaria y en Educación Superior y Tecnologías de la Información y la Comunicación aplicadas a la innovación docente en la Didáctica de las Ciencias Experimentales y en los Prácticum I y II. 